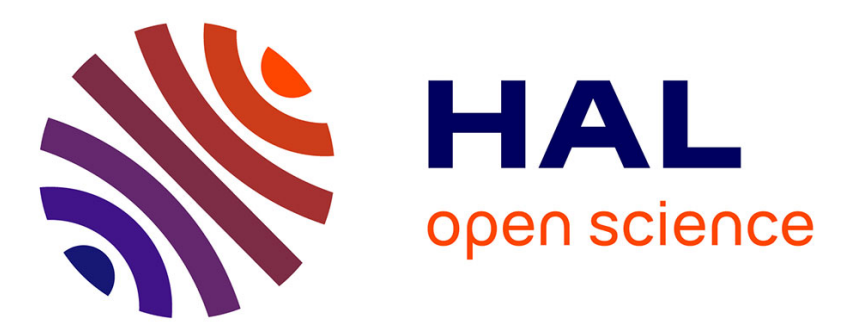

\title{
New possibilities of diffraction topography at third generation synchrotron radiation facilities
}

\author{
F. Zontone, J. Baruchel, J. Härtwig
}

\section{To cite this version:}

F. Zontone, J. Baruchel, J. Härtwig. New possibilities of diffraction topography at third generation synchrotron radiation facilities. Journal de Physique IV Proceedings, 1994, 04 (C9), pp.C9-423-C9430. 10.1051/jp4:1994970 . jpa-00253533

\section{HAL Id: jpa-00253533 \\ https://hal.science/jpa-00253533}

Submitted on 1 Jan 1994

HAL is a multi-disciplinary open access archive for the deposit and dissemination of scientific research documents, whether they are published or not. The documents may come from teaching and research institutions in France or abroad, or from public or private research centers.
L'archive ouverte pluridisciplinaire HAL, est destinée au dépôt et à la diffusion de documents scientifiques de niveau recherche, publiés ou non, émanant des établissements d'enseignement et de recherche français ou étrangers, des laboratoires publics ou privés. 


\title{
New possibilities of diffraction topography at third generation synchrotron radiation facilities
}

\author{
F. Zontone, J. Baruchel and J. Härtwig
}

ESRF, BP. 220, 38043 Grenoble, France

\begin{abstract}
Diffraction topography is an imaging technique for single crystals which allows, through the mapping of the local Bragg reflecting power, the visualization of defects, domains, phases,..., present within the crystal volume. We describe some of the new possibilities of this technique associated with the third generation synchrotron radiation machines: real time observations in the 10-2 second range, investigation of heavy or bulky materials, and high resolution experiments. These new possibilities are illustrated by the first diffraction topographic observations performed at the ESRF, which were carried out, in white beam, either on the wiggler of the ID11 'Materials Science' beamline or on the D5 Optics 'Open Bending Magnet' beamline. The main features of these topographs are the short exposure times $\left(\approx 10^{-2} \mathrm{~s}\right)$, the high energy corresponding to the recorded Bragg spots $(\approx 50-100 \mathrm{keV})$, and the good spatial resolution retained even when setting the film far $(\approx 60 \mathrm{~cm})$ from the sample. We finally briefly describe the ESRF ID19 beamline devoted to high resolution diffraction and topography.
\end{abstract}

\section{INTRODUCTION}

High resolution X-ray diffraction topography [1] was developed during the late fifties and sixties partly in connection with the need to characterize single crystals produced for the electronics industry. The possibilities of the technique were extended to other kinds of single crystal materials, and greatly enhanced when synchrotron radiation was available.

Diffraction topography maps, in real space (as opposed to reciprocal one), the spatial variations of the reflecting power of a single crystal. These variations are usually associated with gradients of distortion related to the presence of defects, domains, phases,... which can be, in this way, visualized. Diffraction topography is non destructive and rather sensitive: distortion gradients associated with an angle $\Delta \theta$ and/or a lattice parameter variation $\Delta \mathrm{d} / \mathrm{d}>10^{-7}$ can be observed. The recording of the diffracted beam is typically performed either on X-ray films or, if the flux is high enough, on video cameras. The detector (film or camera) is set close to the crystal in order to retain the spatial resolution. The crystal to detector distance is $<2 \mathrm{~cm}$ when working with an X-ray generator, and is usually in the $5-20 \mathrm{~cm}$ range when performing white beam diffraction topography (WBDT) at a synchrotron radiation facility. The film resolutions range from 0.1 to several microns and that of video cameras is $\approx 10-50 \mu \mathrm{m}$. The typical exposure time ranges between $0.5-5$ hours when using a rotating anode, and 1-100 seconds at a synchrotron radiation facility. This exposure time reduces to about $10^{-2}-10^{-1} \mathrm{~s}$ when working at a third generation synchrotron facility like the ESRF.

The image associated with a given crystalline defect is produced by regions which are distant from the core of the defect. This distance does not only depend on the nature of the defect, but also on the diffraction process itself. The defect related lattice distortion acts on diffraction through an effective misorientation $\eta$, which reflects the departure from the Bragg angle associated with the existence of the defect. It has been shown [2] that: 


$$
\eta=-\left(\lambda \sin 2 \theta_{B}\right)\left(\mathrm{d}(\mathbf{h} \cdot \mathbf{u}) / \mathrm{d}\left(\mathrm{s}_{\mathrm{h}}\right)\right)
$$

where $\theta_{B}$ is the Bragg angle, $\lambda$ the wavelength of the diffracted radiation, $\mathbf{h}$ the undistorted reciprocal lattice vector, $u$ the displacement vector, and $d / d\left(s_{h}\right)$ represents the derivative along the reflected beam direction. In the low absorption case, which is the only one considered in the present paper, the image is mainly produced by regions surrounding the core of the defect for which the effective misorientation $\eta$ is about twice the intrinsic reflection width $\omega_{\mathrm{D}}$ ("Darwin width") with respect to the matrix 'perfect' crystal [3]. In the symmetrical transmission case

$$
\omega_{\mathrm{D}}=\left(2 \lambda^{2} \mathrm{r}_{\mathrm{c}}\left|\mathrm{F}_{\mathrm{h}}\right|\right) / \pi \mathrm{V}_{\mathrm{c}} \sin 2 \theta_{\mathrm{B}}
$$

where $F_{h}$ is the structure factor, $r_{c}$ the classical radius of the electron, and $V_{c}$ is the unit cell volume. The typical values for $\omega_{D}$ are in the arc second range. This leads to 'intrinsic' image width of a few microns, which is the actual limit of the spatial resolution on the topograph, and implies that rather perfect crystals (with, for instance, a dislocation density $<10^{6} \mathrm{~cm} / \mathrm{cm}^{3}$ ) are required in order to be able to observe individual defects.

We describe in the present paper some of the enhanced new possibilities of the diffraction topographic techniques (real time, heavy or bulky materials, high resolution) offered by the third generation synchrotron radiation machines. These possibilities are partly illustrated by recent test experiments performed on the ID11 Wiggler 'Materials Science' beamline [4] and the D5 'Optical' Open Bending Magnet beamline of the ESRF. We indicate in addition some of the required conditions to carry out a WBDT experiment. Finally we report about the present state of the ESRF ID19 'Topography and High Resolution Diffraction' beamline, and the instrumentation designed to optimize the experimental conditions.

\section{REAL TIME EXPERIMENTS}

A 'real time' experiment is such that the recording time of the pertinent information is shorter than the typical evolution time of the physical process. The early high resolution topographs required several hours of exposure time, and consequently were only used to investigate statical or quasistatical phenomena. The availability of synchrotron radiation for materials scientists, through the reduction of the exposure times to a few seconds, allowed investigation, in 'real time', of a range of physical processes. A wide variety of these kind of investigations is presently being produced on defect movements, resonator vibrations, domains and phases transitions, crystal growth,... .

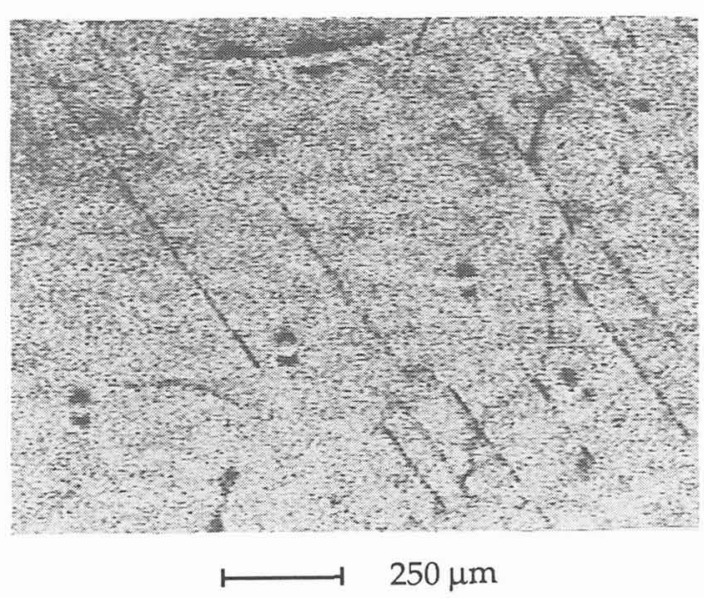

Fig. 1 Enlargements of the $11 \overline{1}(\lambda \approx 0.26 \AA)$ topograph of the germanium crystal $0.325 \mathrm{~mm}$ thick.

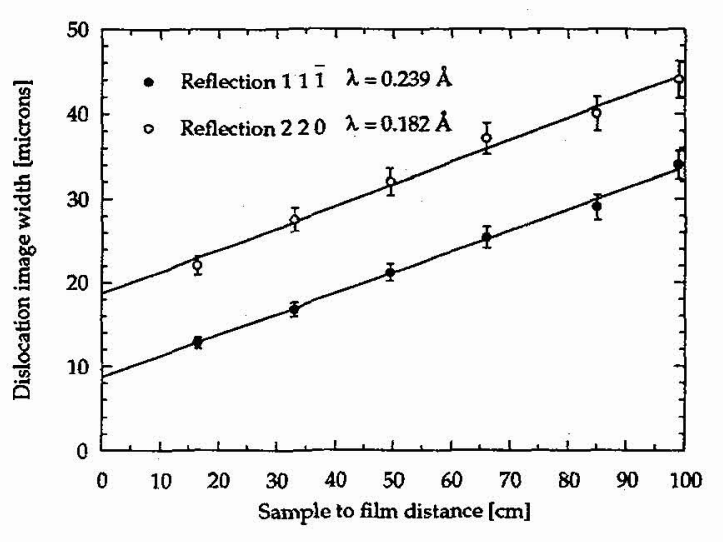

Fig. 2 Image width vs sample-to-film distance of the central screw dislocation of Fig.1. The calculated kinematical image widths are $8.7 \mu \mathrm{m}$ for the 111 and $18.6 \mu \mathrm{m}$ for the 220 reflections respectively. 
Figs. 1 and 2 illustrate the diffraction topographic 'real time' new possibilities when working at the ESRF. Fig. 1 is a $11 \mathrm{~T}$ white beam topograph of a $0.32 \mathrm{~mm}$ thick (T10) germanium crystal, where dislocations and inclusions can be observed. This topograph corresponds to a wavelength $\approx 0.26 \AA$ (48 $\mathrm{keV}$ ), and was recorded in only $10^{-2}$ second when using the ID11 wiggler as a source. Fig. 2 shows the evolution (measured on D5) of the dislocation width as a function of the distance.

These results indicate that 1 ) the X-ray background and the heating of the sample can be reduced to a level such that high quality images can be produced with standard film material 2) very short exposure times (down by about a factor $10^{2}-10^{3}$ below the standard exposure time at other synchrotron radiation topographic setups) can be achieved, and 3) the width of the dislocations extrapolated to zero distance, are in perfect agreement with the 'intrinsic' calculated ones $(8.7 \mu \mathrm{m}$ for the $11 \mathrm{~T}$ reflection, and $18.6 \mu \mathrm{m}$ for the 220 one), and the slope $\left(2.510^{-5}\right)$ of the fig. 2 line is small enough to retain a good spatial resolution when setting the film as far as 1 meter from the sample. This last feature corresponds to the fact that the source is seen, from one point of the sample, under a very small angle. Very often the investigated dynamical phenomena result from the variation of an applied external parameter (temperature, field, stress,...) which implies a bulky sample environment (furnace, cryostat, electromagnet, traction device machine,...), and consequently an enhanced crystal to detector distance. To retain a good resolution under these conditions is therefore practically important.

White beam diffraction topography is usually foreseen when planning 'real time' experiments. This is even more necessary for crystal growth investigations, where the orientation of the sample is not known in advance. WBDT amounts to the usual Laue method, but the weak angular source size has the effect that every Bragg spot is a topograph. WBDT produces of course several diffraction topographs simultaneously and usually reduces the exposure times with respect to its monochromatic counterpart. When considering the flux available from an insertion device at the ESRF, it could be thought that exposure times could be reduced to the few microseconds level. This is unfortunately not the case. Indeed the inhomogeneous heat load associated with the absorbed power can produce an unacceptable distortion of the sample, the topograph becoming meaningless. This is often the case because the beam is small (some $\mathrm{mm}$ ) in the vertical direction, and because the higher energies are in addition concentrated in its center. The non uniform distribution of the absorbed power density $\mathrm{P}_{\mathrm{abs}}$ along the vertical direction creates a gradient of temperature, and consequently of distortion which is observed on the topographs. Fig. 3a to $\mathrm{c}$ is an example which shows the same topograph recorded under different filtering conditions of the beam. The observed contrast can be roughly explained by the simple model depicted in Fig. 3d. The bump which is generated does not affect, for symmetry reasons, the Bragg planes in the middle part of the illuminated area but distorts the outer areas and consequently produces a large amount of additional intensity on the topograph.

This model also suggests a criterion for fixing the maximum absorbed power which does not affect the image. It has been derived, starting from the steady state differential equation for the lateral heat flow in the thin plate case, and under the assumptions that the incoming power displays a gaussian distribution of root mean square $\sigma$ centered around $z=0$, and that no temperature difference is present between the front face and the back one. This last assumption is a reasonable one when the critical energy of the source is $>20 \mathrm{kev}$, as is often the case at the ESRF. The criterion is based on the physical idea that the image at the $z$ position $(|z|<\sigma$ ) will be a good one if the angular variation $2 \Delta$ at this position (fig. $3 d$ ) is less than the Darwin width $\omega_{\mathrm{D}}$ of the considered reflection. This idea adopts, after calculations, a very simple mathematical expression:

$$
2 \Delta / \omega_{\mathrm{D}}<1 \text { with } 2 \Delta=\sqrt{\pi} \alpha \mathrm{P}_{\mathrm{abs}}|\mathrm{z}| / \kappa
$$

where $\alpha$ is the linear thermal expansion coefficient, and $\mathrm{K}$ is the thermal conductivity of the sample.

Table 1 shows the values for $2 \Delta / \omega_{D}$ at $|z|=\sigma(=1 \mathrm{~mm}$ in our case), as well as the calculated incoming and absorbed power densities, corresponding to Fig. 3 topographs. When $2 \Delta / \omega_{\mathrm{D}}$ is 0.85 (Fig. $3 \mathrm{c})$, the horizontal dark-white-dark heat load associated stripes are not observed any more.

Table 1

\begin{tabular}{|c|c|c|}
\hline $\begin{array}{c}\text { Incoming Power density } \\
\mathrm{W} / \mathrm{mm}^{2}\end{array}$ & $\begin{array}{c}\text { Absorbed Power density } \\
\mathrm{W} / \mathrm{mm}^{2}\end{array}$ & $2 \Delta / \omega_{\mathrm{D}}$ \\
\hline \hline 0.85 & 0.080 & 11 \\
\hline 0.31 & 0.015 & 1.75 \\
\hline 0.21 & 0.008 & 0.85 \\
\hline
\end{tabular}


The reported test experiments indicate that 'real time' (in the $10^{-2}-10^{-1} \mathrm{~s}$ range) diffraction topographic high resolution investigations under a bulky sample environment are immediately possible on several ESRF lines, but that they imply some precautions in order not to distort the sample. When working with the white beam from a wiggler or a bending magnet, filtering of the beam appears necessary. The best filter for a given material is the material itself. As this is not always feasible, a 'liquid' filter containing the heaviest elements present in the investigated crystal appears to be a good solution. This was recently confirmed by a test investigation on $\mathrm{D} 5$ on $\alpha-\mathrm{LiIO}_{3}$ crystals where an aqueous solution of potassium iodide was set, as a filter, upstream in the beam, and improved substantially the recorded topographs.

Among the new experiments we plan to perform, let us mention the dislocation propagation under high stresses, the study of domains in magnetic materials close to phase transitions to identify the role of domains and interfaces in critical phenomena, and crystal growth in-situ to investigate the role of defects in the growth process and the mechanism of defect formation.

\section{INVESTIGATION OF HEAVY OR BULKY CRYSTALS}

Another particular feature illustrated by the first diffraction topographic test experiments performed at the ESRF [4] is the high energy corresponding to the recorded Bragg spots $(\approx 50-100 \mathrm{keV})$. This allows the high resolution investigation of bulky or heavy samples (heavy semiconductors, rare earth compounds,...). Recent experiments provide two examples of these new possibilities: the investigation, in transmission, of a $1 \mathrm{~cm}$ thick silicon and of a $1.4 \mathrm{~mm}$ thick iron crystals.

a)

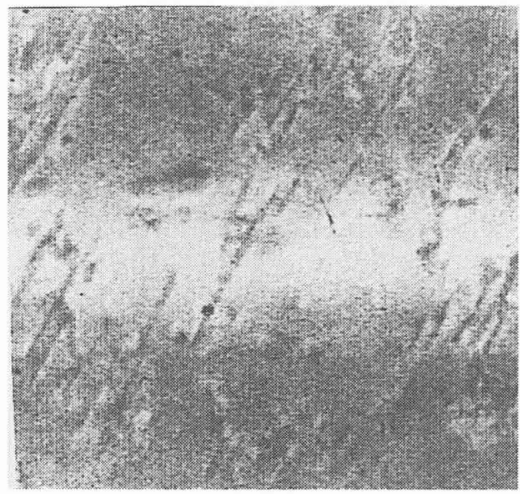

$$
\mathrm{P}_{\mathrm{abs}}=0.080 \mathrm{~W} / \mathrm{mm}^{2}
$$

c)

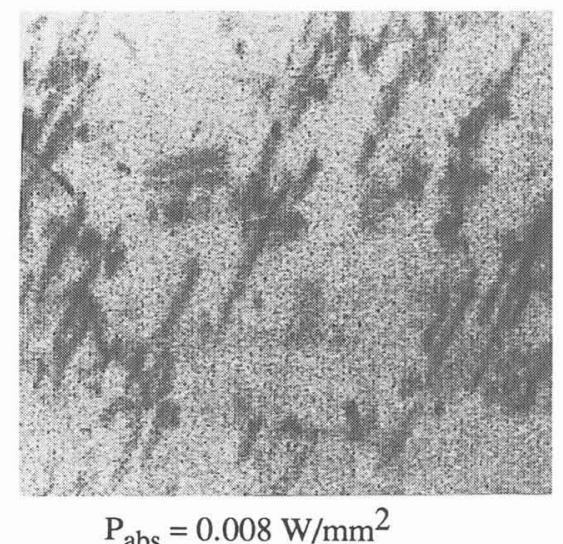

b)

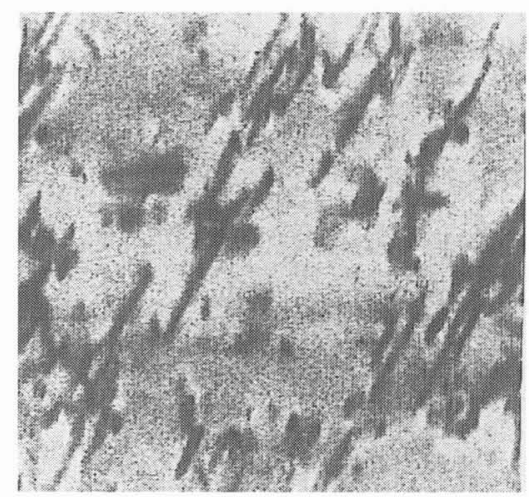

$0.5 \mathrm{~mm}$

$$
\mathrm{P}_{\mathrm{abs}}=0.015 \mathrm{~W} / \mathrm{mm}^{2}
$$

d)

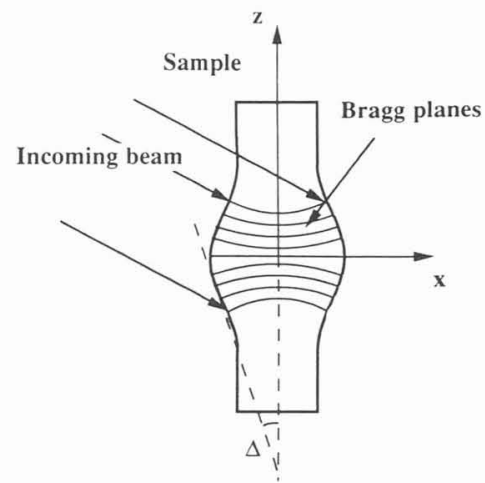

Fig. 3 The heat load effect on the topographs of the Germanium crystal (fig. 4a,b,c) for different absorbed power densities (reflection $111, \lambda \approx 0.493 \AA, E \approx 25 \mathrm{keV}$ ), and the model used for explaining the contrast (fig. $4 \mathrm{~d}$ ). Due to the filtering, the images of fig. $4 \mathrm{~b}$ and $4 \mathrm{c}$ are related to the 333 reflection. 
Fig. 4 shows a 'section' topograph of a $1 \mathrm{~cm}$ thick Si crystal (1T̄0) entrance and exit surfaces, 111 reflection, $\lambda \approx 0.21 \AA, E \approx 59 \mathrm{keV}, \mu \mathrm{t} \approx 0.7$, crystal to image distance $\approx 14 \mathrm{~cm}$, obtained by restricting the vertical beam width to $\approx 20 \mu \mathrm{m}$. As the Bragg angle was only $\approx 3.7^{\circ}$, the film was set nearly parallel to the direct transmitted beam in order to obtain a reasonable image width. The image displays Kato's Pendellösung fringes [5], which indicate the high crystalline quality of the sample. Some other lines just correspond to surface scratches on the exit surface.

Fig. 5 shows the $\overline{2} 00$ topograph $(\lambda \approx 0.146 \AA, E \approx 85 \mathrm{keV}, \mu \mathrm{t} \approx 0.57)$ of a $1.4 \mathrm{~mm}$ thick (001) $\mathrm{Fe}-3 \mathrm{wt} \% \mathrm{Si}$ crystal. Subgrains, with size around $1 \mathrm{~mm}$, are clearly visible through the orientation contrast at their boundaries, and magnetic domains (the classical Landau and Lifshitz domain structure) are revealed in the form of lines parallel to [010]. The domains are visible through magnetostriction, particularly at the "Y junction" lines [6] where the $90^{\circ}$ walls of the closure domains meet the main $180^{\circ}$ walls.

The X-ray topographic observation of similar features (Kato's fringes, domains,...), in transmission, was achieved in the past on samples 10 times thinner. The thick or heavy samples were, up to now, the exclusive domain of (low resolution) neutron diffraction topography [1].

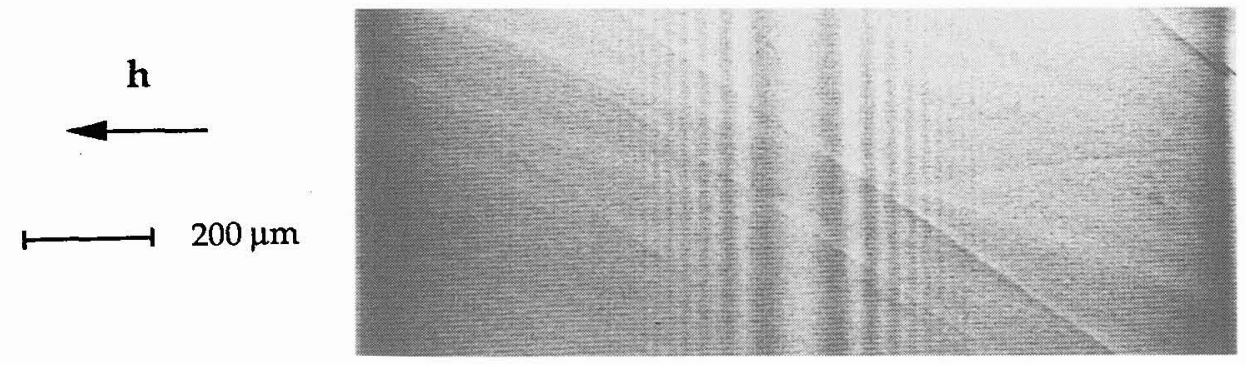

Fig. 4 'Section' topograph of a $1 \mathrm{~cm}$ thick silicon crystal, 111 reflection $(\lambda \approx 0.21 \AA, E \approx 59 \mathrm{keV})$ crystal to image distance $\approx 14 \mathrm{~cm}$, vertical beam width restricted to $\approx 20 \mu \mathrm{m}$.
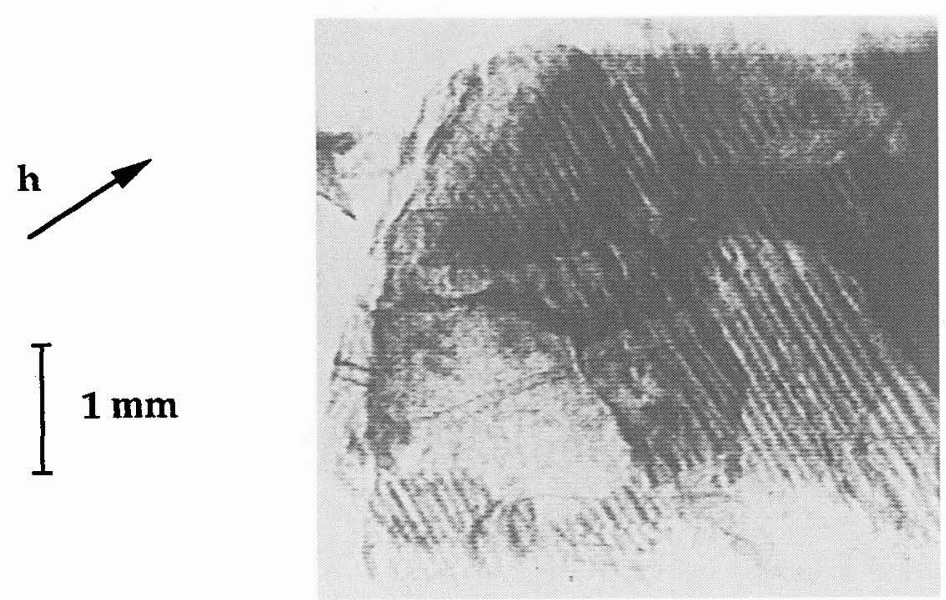

Fig. 5 The $\overline{2} 00$ topograph $(\lambda \approx 0.146 \AA, E \approx 85 \mathrm{keV})$ of a $1.4 \mathrm{~mm}$ thick (001) Fe-3\%Si crystal. 


\section{HIGH RESOLUTION DIFFRACTION TOPOGRAPHIC EXPERIMENTS}

The expression 'High resolution diffraction topographic experiments' is found in the literature to describe at least two distinct kinds of experiments: either those where a very high spatial resolution is achieved, or the ones where a very high sensitivity to weak gradients of distortion is required (plane wave or 'ultra'-plane wave topography). Both types of experiment will benefit from the third generation synchrotron radiation possibilities, through the high flux, the available high energy $(\approx 50-100 \mathrm{keV})$ photons, and the good angular resolution associated with the small size of the source and the big distance between this source and the sample.

The enhanced flux means that images can be formed from weaker scattering processes. This will allow exploitation of weak beam topography, which leads to narrower defect images, hence raising the density at which individual defects can be resolved. Another interesting possibility, which is not connected with high resolution, is the use of resonant magnetic scattering to perform images of magnetic domains using a contrast mode arising directly from the magnetization or the magnetic arrangement.

Fig. 6 illustrates the effects associated with high energy photons. This $11 \overline{2} 1$ topograph of a $6 \mathrm{H}-$ $\mathrm{SiC}$ crystal, produced with $59 \mathrm{keV}$ photons, exhibits double contrast dislocation images. We observed very frequently this feature, already reported in special cases in the past [7]. It is related to the fact that the Darwin width $\omega_{\mathrm{D}}$ decreases when the photon wavelength does, and consequently the regions which are at the origin of the image correspond to very weak deformations (of the order of $\omega_{\mathrm{D}}$ ) with respect to the perfect crystal. The use of high energies is therefore a simple way to evidence these very weak gradients of distortion. Among the possibilities, the investigation of microdefects in semiconductors could be particularly interesting in the crystals presently produced for the microelectronics industry. The strain field of these microdefects could be fully investigated using a monochromatic beam produced by a single monochromator (as opposed to channel cut or multiple crystal one). Indeed the angular width corresponding to the source size divided by the source-to-monochromator distance is usually smaller than the monochromator Darwin width, producing in this way a quasi-plane wave.
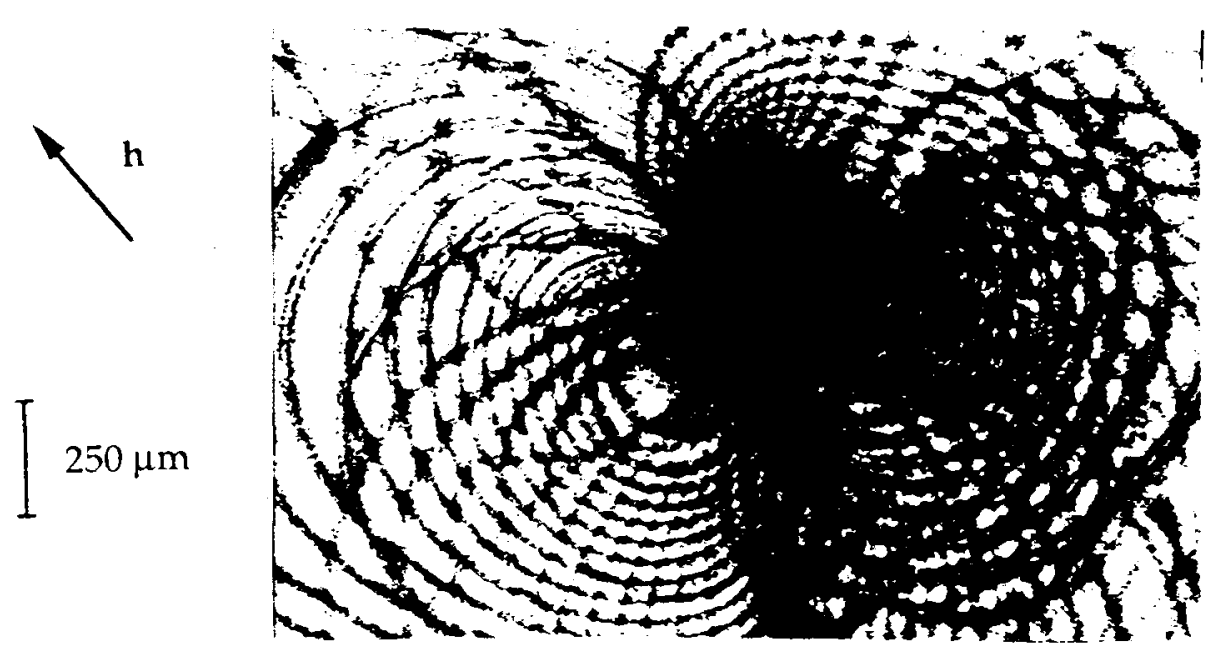

Fig. 6 Network of dislocations in the $11 \overline{2} 1$ topograph $(\lambda \approx 0.21 \AA, E \approx 59 \mathrm{keV})$ of a $6 \mathrm{H}-\mathrm{SiC}$ sample. 


\section{THE DIFFRACTION TOPOGRAPHY ESRF BEAMLINE}

The ESRF ID19 'Topography and High Resolution Diffraction' beamline is expected to be fully operational in autumn 1995. Its main features derive from the requirements of having a spectrally and spatially homogeneous beam at the sample position, with dimensions $\approx 40 \times 14 \mathrm{~mm}^{2}$, a high photon flux and a tunable photon energy in the range $6-120 \mathrm{keV}$. These requirements lead to the choices of a long $(145 \mathrm{~m})$ beamline, and an eleven poles, variable gap, high-magnetic field wiggler $\left(\mathrm{B}_{\max }=1.5 \mathrm{~T}\right)$, $\mathrm{K}_{\max }=21, \mathrm{E}_{\mathrm{c}, \max }=35 \mathrm{keV}$ ) as source [8].

The optical hutch, located just after the front end, will be ready in december 94 . It will mainly contain the diaphragms, slits, and filters It will be possible to perform, in this hutch, experiments requiring a small $\left(\approx 10 \times 4 \mathrm{~mm}^{2}\right)$ but very intense beam. An about $100 \mathrm{~m}$ long tunnel will join the first hutch to the satellite laboratory (already built), outside the experimental hall. The monochromators and experiments hutches will be constructed in this satellite laboratory in september 94 . The experimental hutch $\left(5 \times 5 \mathrm{~m}^{2}\right.$, and $4 \mathrm{~m}$ height) will have a marble floor such that equipment could easily be transferred, and new optical element mounts quickly be made up.

The beamline is designed to use either the white beam or a monochromatic beam in the experiments hutch. The monochromatic beam will be delivered, as a function of the required $\Delta \mathrm{E} / \mathrm{E}$, by two types of monochromators. The first one, located in the experiments hutch, consists of a 'single' crystal diffracting in the horizontal plane. In addition, it will be possible to vibrate this monochromator, up to $200 \mathrm{~Hz}$, within a small $(\approx 0.5$ mradians) angular range. This allows to overcome the spatial energy dispersion associated with the extended beam surface and the small source size $(0.2 \mathrm{~mm})$, which is a problem for some applications. The second monochromator will be a 'Hasylab type' double crystal, fixed exit, one. It will diffract in the vertical plane and be located in the monochromators hutch. As mirrors cannot be used because even the best ones produce a rather inhomogeneous reflected beam [9], the first crystal will be water cooled. This double crystal monochromator can be combined with another monochromator in the experimental hutch to produce an 'ultra plane wave', which is required for special applications.

We are presently commissioning a "sample environment" setup for 'real time' (white beam or monochromatic) experiments, which moves on a marble floor. It accomodates special stages including a liquid helium or a closed cycle helium cryostats, an electromagnet, a high temperature furnace and a straining stage (designed in collaboration with colleagues from the CNRS-Marseille and Nancy-). On the other hand a "high precision" camera is being designed (again in collaboration with experienced colleagues from the CNRS -Paris-) to optimize the angular and spatial resolution. Special slits are being built for 'section' and 'pinhole' diffraction topography. A shutter operating in the millisecond timescale was developed.

Detectors are a crucial part of the diffraction topography instrument. A substantial effort is being devoted to this topic, in order to make the best possible use of the ESRF source. Several kinds of detectors are currently used: CCD cameras equiped with a visible light optics and scintillators, films, energy sensitive proportional counters. The detection is also coupled with specific image treatments adapted for the diffraction topography techniques with this source. Two kinds of image treatment are being considered: on line processing and post acquisition treatment. Simple processing to decrease the noise in the image, or to substract images, will be available. Post acquisition treatments includes various types of filtering. A combination of commercial software and specially written programs (collaboration with the University Paris VI, which also includes the sophisticated diffraction topographic image simulation programs) will cover our needs.

\section{SOME CONCLUSIONS}

Novel experiments are possible, using the diffraction topographic techniques at third generation synchrotron radiation facilities. This is confirmed by the first test experiments, which.indicate that the $\mathrm{X}$ ray background level within the hutch, and the heating of the sample, can be reduced to such a level that white beam topography with standard film material is possible on most of the samples.

The main, and very encouraging, features of these topographs are:

a) the short exposure times $\left(\approx 10^{-2}-10 \mathrm{~s}\right.$, a factor $10^{2}-10^{3}$ below the standard exposure time at other synchrotron radiation topographic setups), due to the high beam intensity;

b) the high photon energy corresponding to the recorded Bragg spots ( up to $\approx 110 \mathrm{keV}$ );

c) the good spatial resolution retained even when setting the film at about $1 \mathrm{~m}$ from the sample, associated with the small source size and large source-sample distance, resulting in very small effective divergence as seen from a point in the specimen, and

d) the images observed on the topographs are in accordance with the presently accepted theory, when taking into account the short wavelength of the radiation and the small size of the source. 
This allows the real time investigation of large or heavy materials under bulky sample environment. On the other hand high energy photons allow to evidence very weak gradients of distortion.

Planned experiments are studies involving dislocation propagation, under high stresses and at high temperature, non-equilibrium shape and transient defects during wall and/or interface movements, and their interactions with the defects present in the crystal, heavy semiconductors, rare earth compounds, and crystal growth of industrial materials.

All the mentioned possibilities, as well as new ones, will be optimized at the ID19 Topography and High Resolution Diffraction beamline (fully operational in autumn 1995). For instance the variable gap of the wiggler will make possible to change the strength of the magnetic field allowing the access to both low and high $\mathrm{X}$-ray energies. Furthermore the increased distance from the source will improve the resolution with respect to the reported preliminary tests. Finally the very large spatial transverse coherence length of the photons $(\approx 100 \mu \mathrm{m})$ associated with the big sample to source distance and the small size of the source $(\approx 0.2 \mathrm{~mm})$ allows to have a large area coherent beam. This opens a promising field of research, the investigation of the speckles in the diffraction pattern corresponding to phase jumps in the crystal. An example of such a diffraction topographic investigation can be the variation as a function of the temperature of the size of antiphase domains.

\section{ACKNOWLEDGMENTS}

We wish to thank all the colleagues who helped us during the first diffraction topographic experiments at the ESRF: Åke Kvick and Michael Krumrey from the Diffraction group, Andreas Freund and Guy Rolland from the Optics Group, and Ray Barrett, Laurent Chabert, René Chagnon, Marc Diot, Ange Draperi, Yves Epelboin, Jo Gastaldi, Jiri Kulda, Eetu Prieur, Petra Rejmankova, Cécile Revol and Michel Schlenker.

\section{REFERENCES}

[1] for a brief review on topographic techniques see BARUCHEL J. in "Neutron and Synchrotron Radiation for Condensed Matter Studies: theory, instruments and methods", HERCULES vol.I, p.399, edited by J. Baruchel, J. L. Hodeau, M. S. Lehmann, J. R. Regnard and C. Schlenker, Ed. de Physique and Springer Verlag (1993), and references therein

[2] AUTHIER A. , Adv. X-ray Analysis, 10, 9 (1967)

[3] MILTAT J. and BOWEN D. K., J. Appl. Cryst. $\underline{8}, 657$ (1975)

[4] BARUCHEL J., EPELBOIN Y., GASTALDI J., HÄRTWIG J., KULDA J., REJMANKOVA P., SCHLENKER M. and ZONTONE F., Phys. Stat. Sol. (a), 141, 59 (1994)

[5] KATO N, and LANG A. R., Acta Cryst. 12, 787 (1959)

[6] MILTAT J. and KLEMAN M., Phil. Mag. 28, 1015 (1973)

[7] KLAPPER H., J. Appl. Cryst., 9,310 (1976)

[8] BARUCHEL J., DRAPERI A., ZONTONE F., J. Phys. D (Appl. Phys.), 26, A9 (1993)

[9] LABERGERIE D., SUSINI J., private communication based on very recent experiments 